\title{
Zn-BTC MOF as adsorbent for iodine uptake and organic dye degradation
}

Abani Sarkar, Amit Adhikary*, Arnab Mandal, Tonmoy Chakraborty, and Debasis Das*

Department of Chemistry, University College of Science, University of Calcutta, 92 A. P. C.

Road, Kolkata 700009, India

E-mail:dasdebasis@2001yahoo.com, aadhikary87@gmail.com

\section{Index}

SI. No.

1. Experimental Section

\section{Contents}

Page No.

S2-S3

\begin{tabular}{|c|c|c|}
\hline 2. & IR-Plot & S3 \\
\hline 3. & Various coordination mode of BTC & S4 \\
\hline 4. & Single crystal XRD structure & S5 \\
\hline 5. & SEM image, reduce form of Zn-BTC & S5 \\
\hline 6. & $\mathrm{~N}_{2}$ Gas adsorption isotherms & S6 \\
\hline 7. & DLS plot & S6 \\
\hline 8. & PXRD plot & S7 \\
\hline 9. & Thermo gravimetric Analysis (TGA) & S7 \\
\hline 10. & Percentage of Iodine uptake with time. & S8 \\
\hline 11. & Photographs of iodine release experiment & S8 \\
\hline 12. & EDX spectrum & S8 \\
\hline 13. & $\begin{array}{l}\text { SEM image of Zn-BTC after completing one cycle of iodine } \\
\text { adsorption. }\end{array}$ & S9 \\
\hline 14. & Solid state UV-Plot & S9 \\
\hline 15. & Kinetics of Photocatalytic Dye degradation study & S10 \\
\hline 16. & $\begin{array}{l}\text { SEM image of Zn-BTC after completing one cycle of (a) RhB } \\
\text { and (b) MEB. }\end{array}$ & S10 \\
\hline 17. & $\begin{array}{l}\text { Photocatalytic degradation of RhB by NMOF } 2 \text { with and } \\
\text { without TBA. }\end{array}$ & S11 \\
\hline 18. & Comparison table & S11-S12 \\
\hline 19. & References & $\mathrm{S} 12-\mathrm{S} 13$ \\
\hline
\end{tabular}




\section{Experimental Section}

\section{Preparation of Sample for DLS Experiment.}

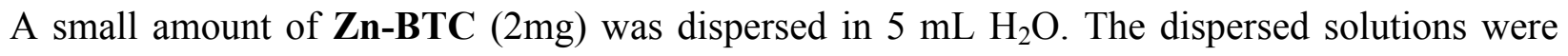
taken in a quartz cuvette and subjected to DLS experiment to get the radius distribution of the solvent coated MOFs.

Titration of the Adsorbed Iodine by Thiosulphate Solution

Preparation of $0.05 \mathrm{M}$ sodium thiosulphate solution.

$250 \mathrm{~mL}$ boiled distilled water was taken in a $500 \mathrm{~mL}$ beaker and $3.1 \mathrm{~g}$ of Sodium thiosulphate $\left(\mathrm{NaS}_{2} \mathrm{O}_{3} \cdot 5 \mathrm{H}_{2} \mathrm{O}\right)$ and few drop of $\mathrm{CHCl}_{3}$ was added in it, and the solution was filtered and marked in a $250 \mathrm{~mL}$ volumetric flask with distilled water.

\section{Preparation of $10 \%$ Potassium iodate solution.}

$5 \mathrm{~g}$ of KI was dissolved on a minimum amount of distilled water and finally it was taken in a $50 \mathrm{~mL}$ volumetric flask and make up the volume with distilled water.

\section{Preparation of $0.05 \mathrm{~N} \mathrm{~K}_{2} \mathrm{Cr}_{2} \mathrm{O}_{7}$.}

Exact $0.245 \mathrm{~g}$ of $\mathrm{K}_{2} \mathrm{Cr}_{2} \mathrm{O}_{7}$ was taken in a $100 \mathrm{ml}$ volumetric flask and make the volume with distilled water.

\section{Standardization of sodium thiosulphate solution.}

Pipate out $25 \mathrm{~mL}$ standared $0.05 \mathrm{~N} \mathrm{~K}_{2} \mathrm{Cr}_{2} \mathrm{O}_{7}$ solution to the $500 \mathrm{~mL}$ conicalflask, added few $\mathrm{mL}$ distilled water and few drop of $\mathrm{H}_{2} \mathrm{SO}_{4}$ to adjust $\mathrm{pH}<0.1$. Added measured excess of $50 \mathrm{~mL}$ iodine solution, and the resultant solution was allow to stand for few second then $1 \%$ starch solution was added to it and the resultante solution was titrate againstSodium thiosulphate solution.

Till the bue colour become discharged.

Standardizations of $\mathrm{Na2S2O3}$ against standard $0.05 \mathrm{~N} \mathrm{~K}_{2} \mathrm{Cr}_{2} \mathrm{O}_{7}$ solution.

Table S1

\begin{tabular}{|c|c|}
\hline Volume of $0.05 N \mathrm{~K}_{2} \mathrm{Cr}_{2} \mathrm{O}_{7}$ solution in $\mathrm{mL}$ & Volume of $\mathrm{NaS}_{2} \mathrm{O}_{2}$ requred \\
\hline $25 \mathrm{~mL}$ & $26.1 \mathrm{~mL}$ \\
\hline \multicolumn{2}{|c|}{$\mathrm{V}_{1} \mathrm{~S}_{1}=\mathrm{V}_{2} \mathrm{~S}_{2}$} \\
\hline \multicolumn{2}{|c|}{$\mathrm{V}_{1}=$ volume of $\mathbf{K}_{\mathbf{2}} \mathbf{C r}_{\mathbf{2}} \mathbf{O}_{7}$} \\
\hline \multicolumn{2}{|c|}{$\mathrm{S}_{1}=$ strength of $\mathbf{K}_{\mathbf{2}} \mathbf{C r}_{\mathbf{2}} \mathbf{O}_{7}$} \\
\hline \multicolumn{2}{|c|}{$\mathrm{V}_{2}=$ volume of $\mathrm{NaS}_{2} \mathrm{O}_{2}$ requred } \\
\hline
\end{tabular}




$$
\begin{gathered}
\mathrm{S}_{2}=\text { strength of } \mathrm{NaS}_{2} \mathrm{O}_{2} \\
\mathrm{~S}_{2}=0.0478
\end{gathered}
$$

\section{Preparation of Zn-BTC@ $\mathbf{I}_{2}$.}

with $50 \mathrm{~g} \mathrm{Zn}-\mathrm{MOF}$, the container was put into a iodine contained biker covered with a watch glass lid and left it for 12 hours on $65^{\circ}$ Cincubator.

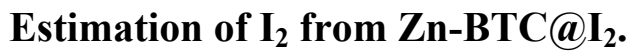

Few mL of ethanol wad added to the prepared Zn-BTC@ $\mathrm{I}_{2}$, then the resultant mixture was taken $2000 \mathrm{~mL}$ volumetric flask and make up the level with distilled water. After 30 minute sonication the $25 \mathrm{~mL}$ of resultant solution was taken in to a conical flask and added $2 \%$ starch solution and the whole solution was titrate against $(0.048) \mathrm{N}$ Sodium thiosulphate $\left(\mathrm{NaS}_{2} \mathrm{O}_{3}\right)$ solution. Found $10.09 \mathrm{~g}$ iodine present in the $50 \mathrm{mg}$ of $\mathrm{Zn}-\mathrm{BTC} \mathrm{MOF}$, i.e $20.18 \%$ iodine has been adsorbed by the Zn-BTC MOF.

$$
\begin{gathered}
\mathrm{I}_{2}+2 \mathrm{~S}_{2} \mathrm{O}_{3}{ }^{2-}=2 \mathrm{I}^{-}+\mathrm{S}_{4} \mathrm{O}_{6}{ }^{2-} \\
\frac{1}{2} \mathrm{I}_{2}=\mathrm{S}_{2} \mathrm{O}_{3}{ }^{2-}
\end{gathered}
$$

Table S2.

\begin{tabular}{|c|c|}
\hline Strength of $\mathrm{NaS}_{2} \mathrm{O}_{2}$ solution(N) & Volume require Strength of $\mathrm{NaS}_{2} \mathrm{O}_{2}$ solution(mL) \\
\hline 0.0478 & 20.8 \\
\hline
\end{tabular}

$$
\begin{aligned}
\text { We know, } & \\
1000 \mathrm{~mL} 1(\mathrm{~N}) \mathrm{Na}_{2} \mathrm{~S}_{2} \mathrm{O}_{3} & =126.9 \mathrm{~g} \mathrm{I}_{2} \quad\left[\frac{1}{2} \mathrm{I}_{2}=\mathrm{S}_{2} \mathrm{O}_{3}{ }^{2-}\right] \\
25 \mathrm{~mL}, 0.048(\mathrm{~N}) \mathrm{Na}_{2} \mathrm{~S}_{2} \mathrm{O}_{3} & =\frac{126.9 \times 0.0478 \times 20.8}{1000} \mathrm{~g} \mathrm{I}_{2} \\
& =0.126 \mathrm{~g} \mathrm{I}_{2}
\end{aligned}
$$

$25 \mathrm{~mL}$ iodine solution contain $0.126 \mathrm{~g} \mathrm{I}_{2}$

2000mL stock iodine solution contains $10.09 \mathrm{~g} \mathrm{I}_{2}$. 


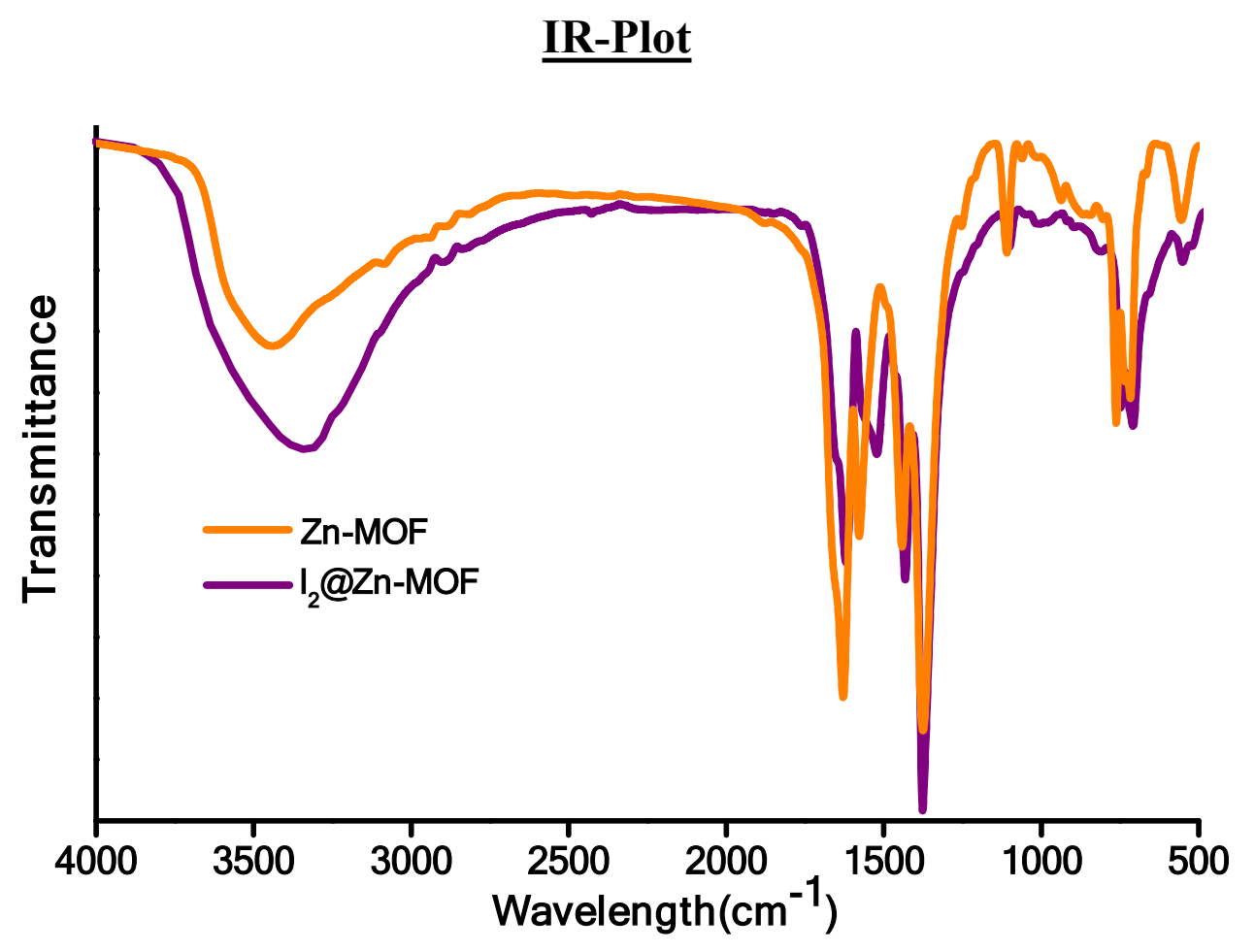

Figure S1. FT-IR spectra of Zn-BTCandI $\mathbf{I}_{2} @ \mathbf{Z n - B T C}$

\section{Coordination mode of BTC}

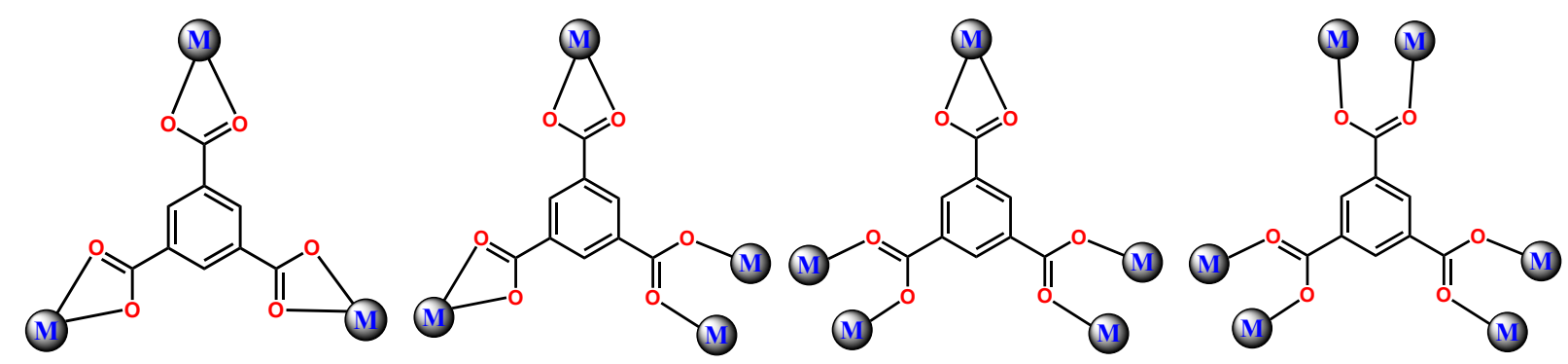
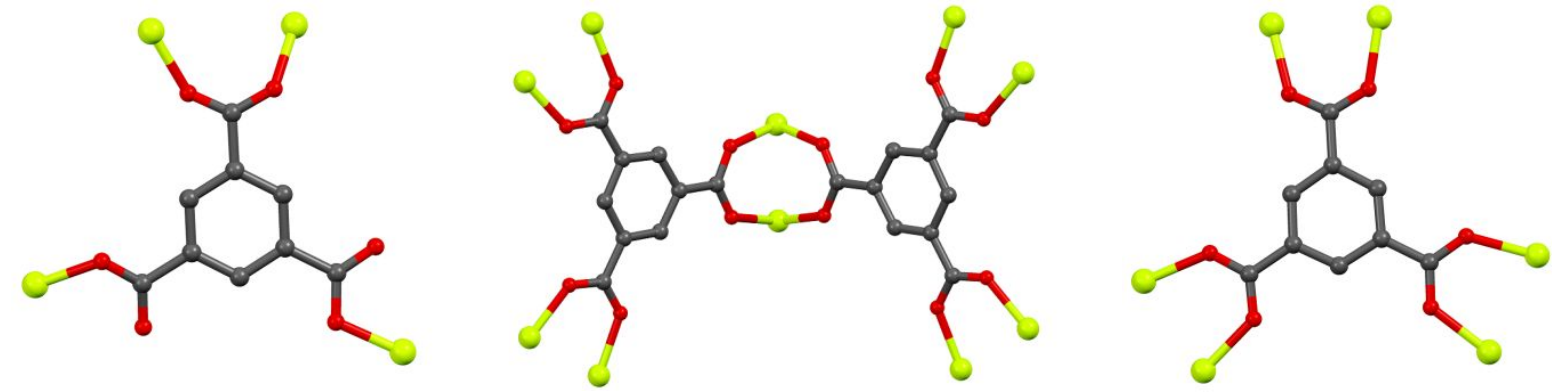

Figure S2. Various coordination mode of $\mathrm{BTC}^{3-1 i g a n d . ~ M: ~ m e t a l . ~}$ 


\section{Single crystal XRD structure}

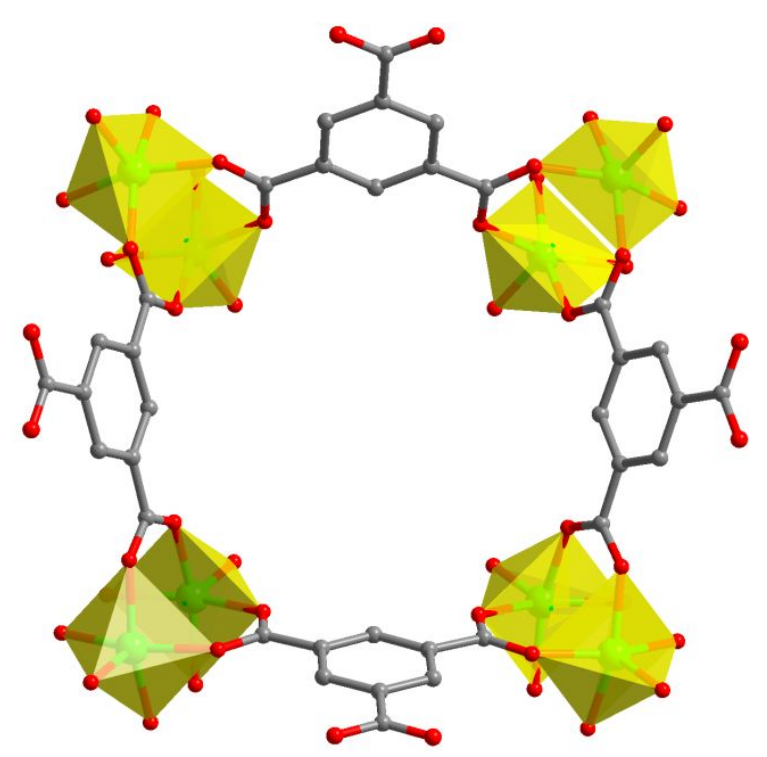

Figure S3. Structural building unit of Zn-BTC.

$\underline{\text { SEM image }}$

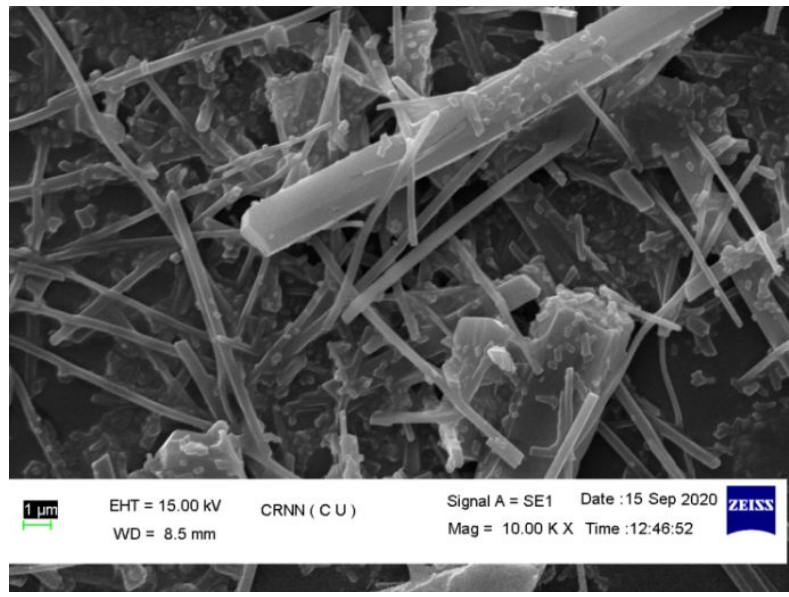

Figure S4. SEM image of grinded crystalline Zn-BTC. 


\section{Gas adsorption isotherms}

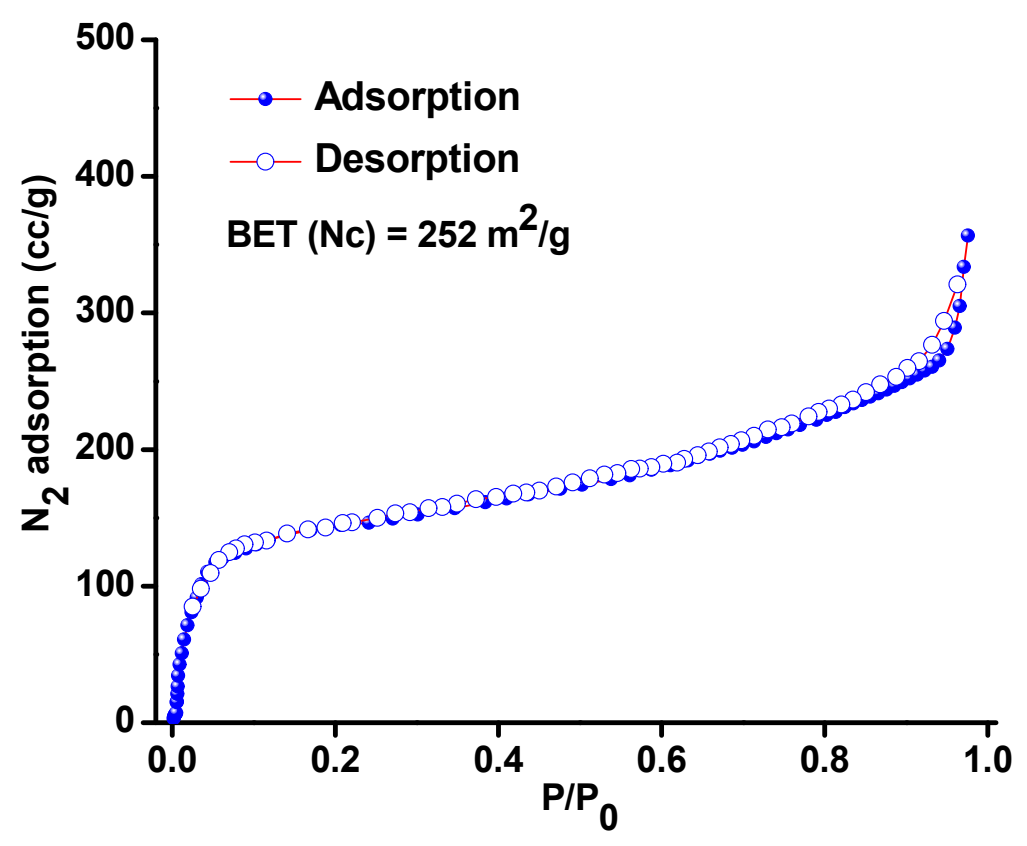

Figure S5. $\mathrm{N}_{2}$ Gas adsorption isotherms of Zn-MOF.

\section{DLS plot}
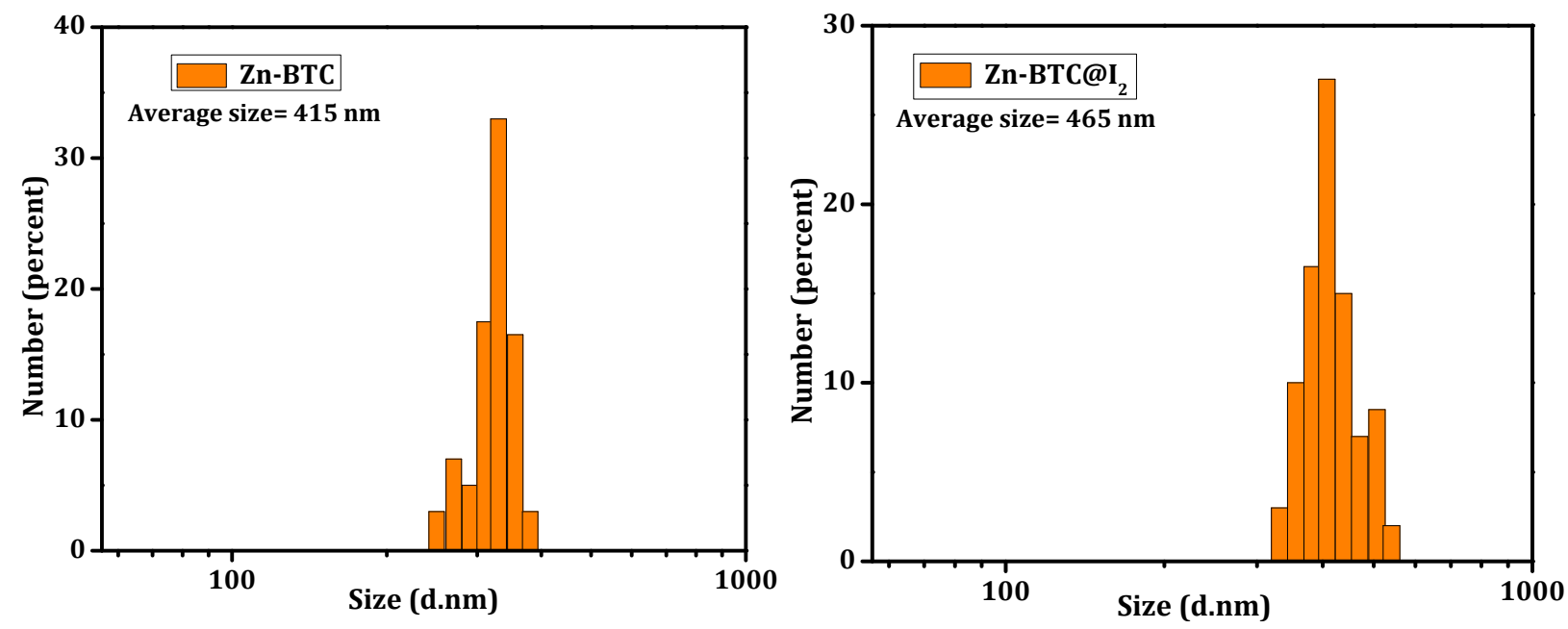

Figure S6. DLS data of Zn-BTCandI $\mathbf{I}_{2} @ Z$ ZnBTC. 


\section{PXRD plot}

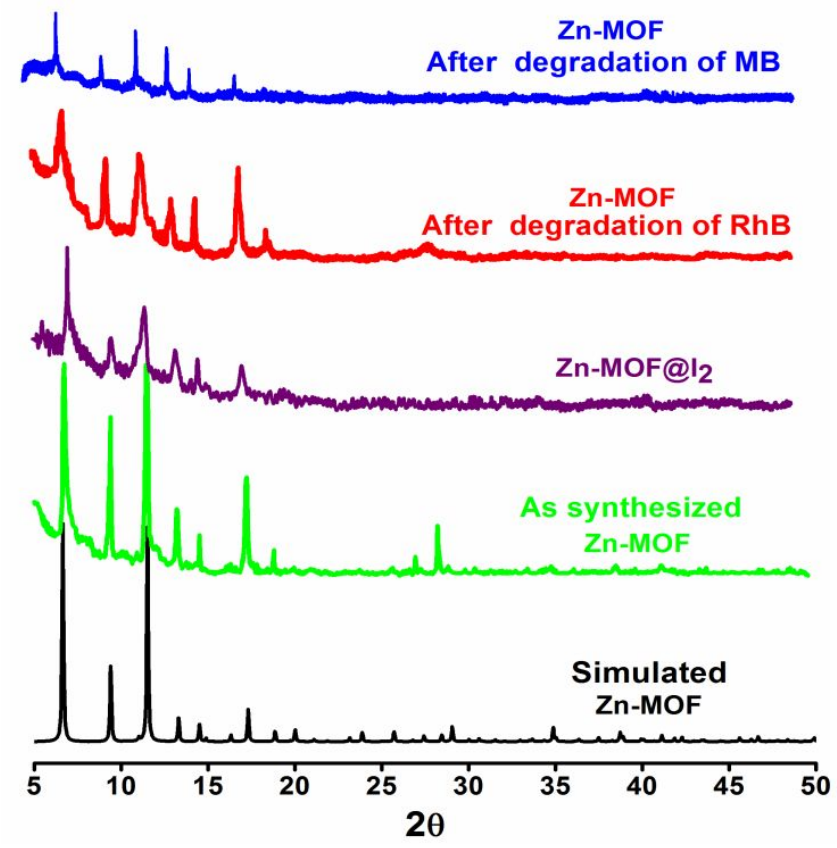

Figure S7. PXRD pattern of simulated, as synthesized Zn-MOF, and $\mathbf{I}_{2} @ Z \mathbf{Z n - B T C}$.

\section{Thermogravimetric Analysis (TGA)}

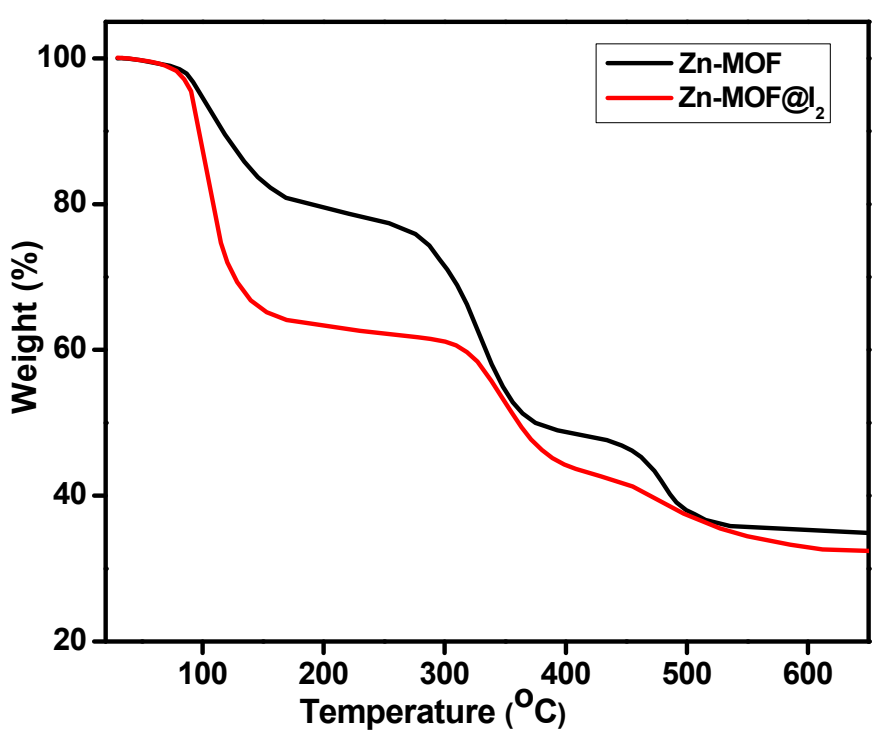

Figure S8. TGA plot of Zn-BTCand Zn-BTC@I 

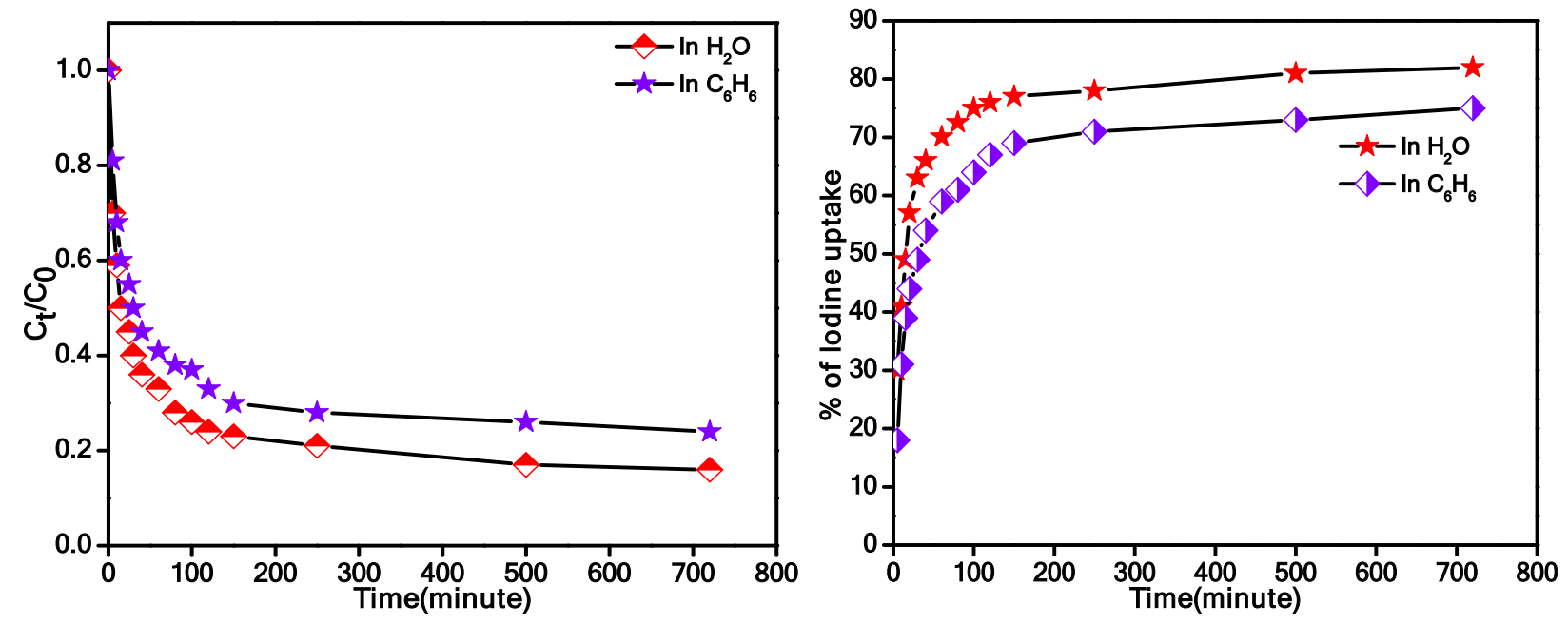

Figure S9. (a) $\mathrm{C}_{\mathrm{t}} \mathrm{C}_{0}$ vs. time plot (b) Percentage of uptake with time.

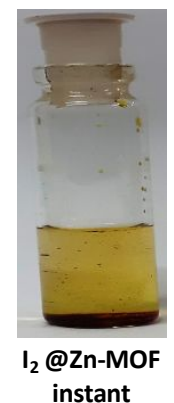

(a)

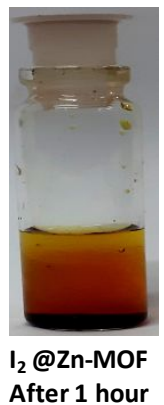

(b)

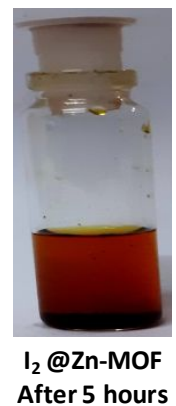

(c)

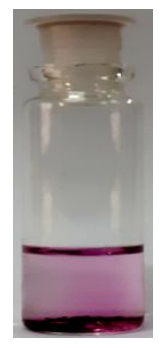

$I_{2} @ Z n-M O F \quad I_{2} @ Z n-M O F$ instant

(d)

(e)

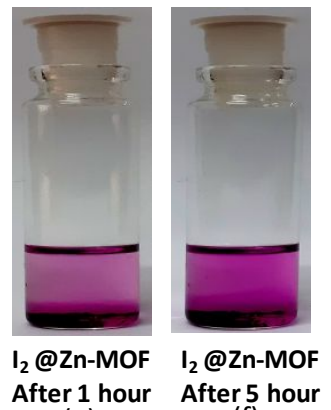

(f)

Figure S10. Photographs of iodine release in aqueous iodide solution and organic hexane solution from $\mathbf{I}_{2} @ Z$ ZnBC.

\section{EDX spectrum}

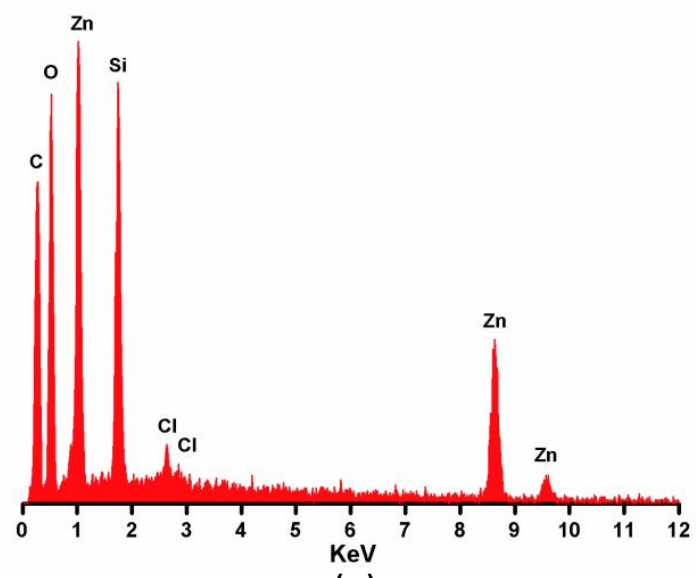

(a)

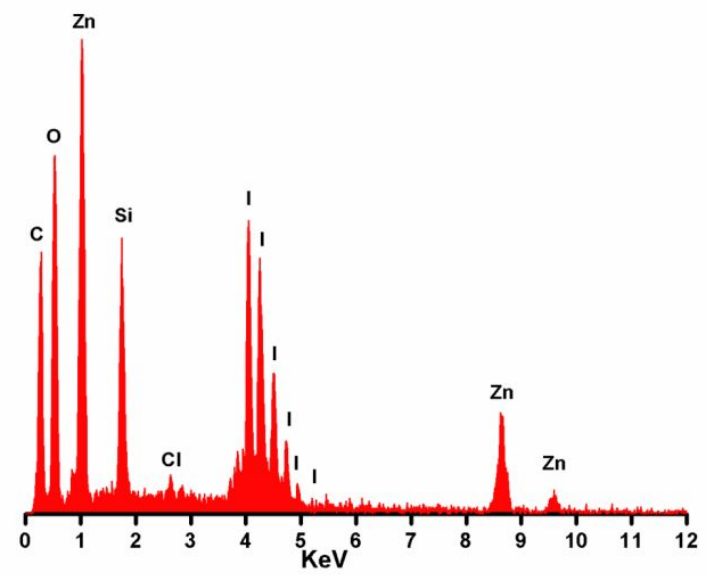

(b) 
Figure S11. EDX plot of (a) Zn-BTC and (b) Zn-BTC@ $I_{2}$.

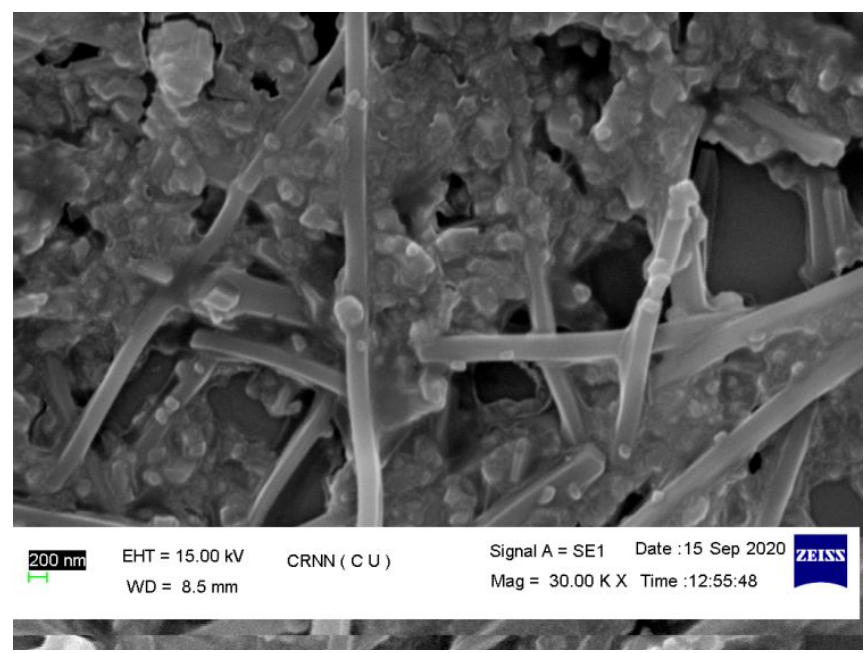

Figure S12. SEM image of Zn-BTC after completing one cycle of iodine adsorption.

\section{Solid state UV-Plot}

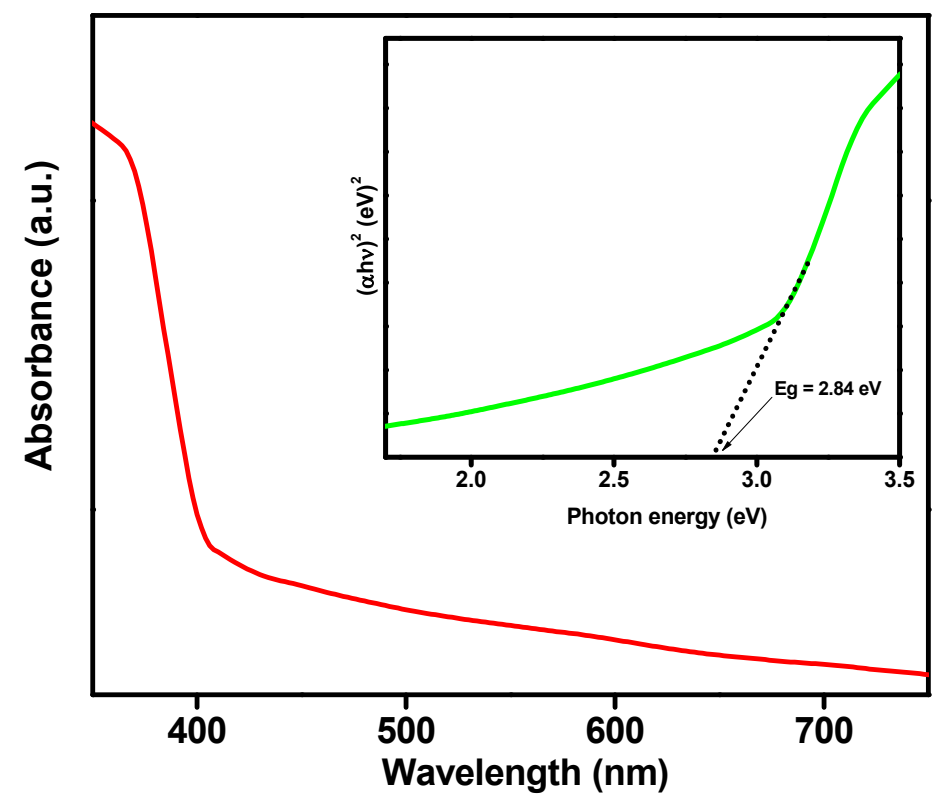

Figure S13. Solid state UV spectra of Zn-BTC. Inset: corresponding Tauc plots of the MOF. 


\section{Photocatalytic Dye degradation study}
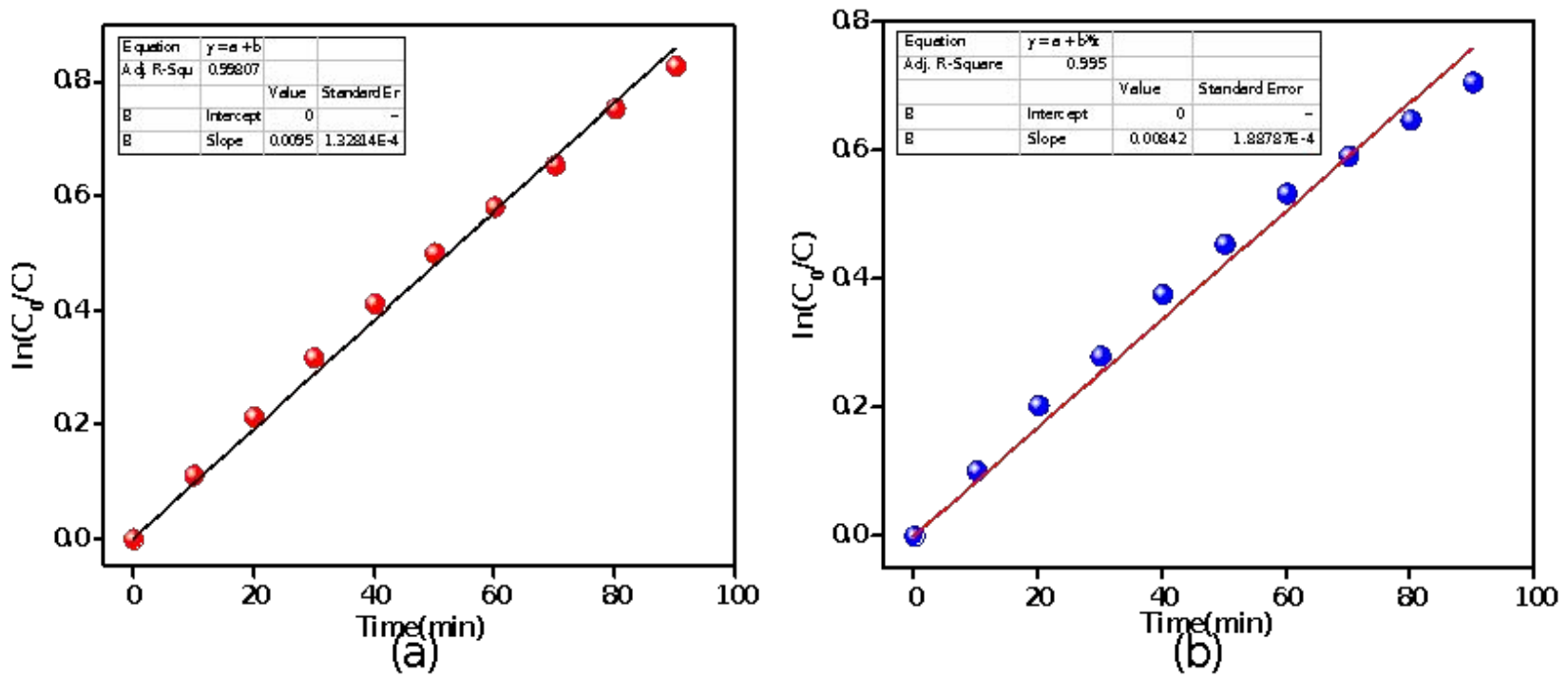

Figure S14. Plot of $\ln \left(C_{0} / C_{t}\right)$ vs time for the pseudo first order kinetics curves of the photocatalytic degradation of (a) MEB and (b) RBH.

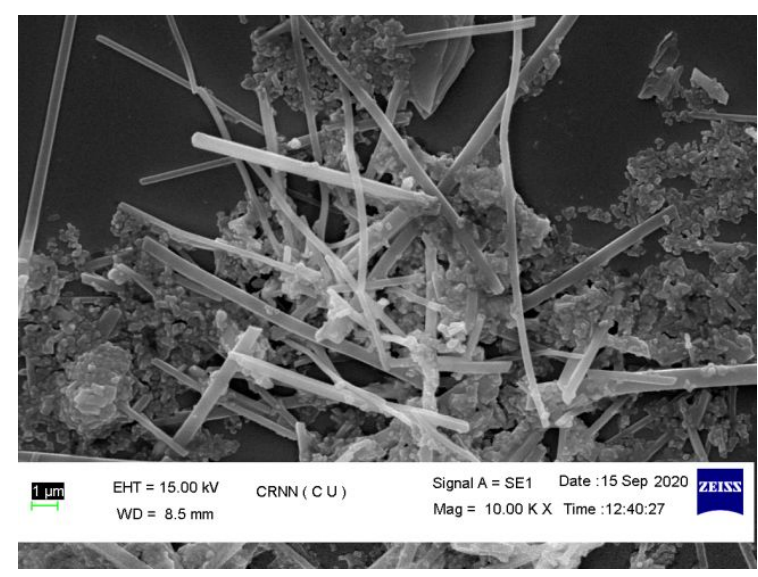

(a)

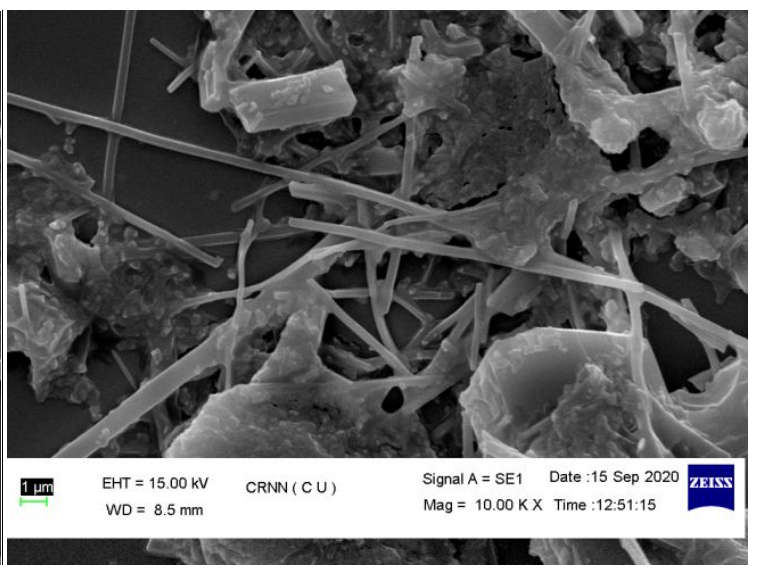

(b)

Figure S15. SEM image of Zn-BTC after completing one cycle of (a) RhB and (b) MEB. 


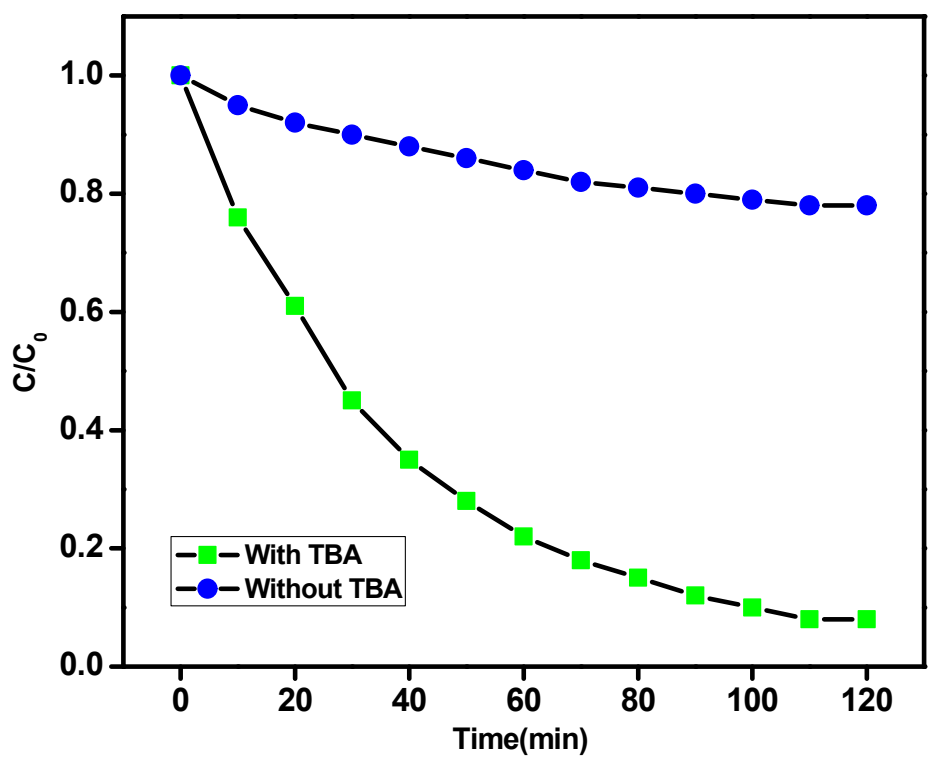

Figure S16. Photocatalytic degradation of RhB by NMOF 2 with and without TBA.

Table S4. Iodine adsorption by some porous MOF previously reported MOFs.

\begin{tabular}{|c|c|c|}
\hline MOF & Efficiency $(\%)$ & Ref. \\
\hline $\mathrm{C}_{22} \mathrm{H}_{17} \mathrm{~N}_{5} \mathrm{O}_{4} \mathrm{Zn}(\mathbf{4 A})$ & $19.65 \%$ & 1 \\
\hline$\left[\left(\mathrm{In}_{3} \mathrm{O}\right)_{2}\left(\mathrm{Cu}_{2} \mathrm{I}_{2}\right)_{3}(\mathrm{ina}) 12\left(\mathrm{H}_{2} \mathrm{O}\right) 6\right]\left(\mathrm{NO}_{3}\right)_{2} \cdot 7 \mathrm{DMA} \cdot 10 \mathrm{H}_{2} \mathrm{O}$ & $45 \%$ & 2 \\
\hline $\mathrm{MII}(\mathrm{pz})[\mathrm{MII}(\mathrm{CN}) 4]\left(\mathrm{M}=\mathrm{Ni}, \mathrm{Co}, \mathrm{M}^{\prime}=\mathrm{Ni}, \mathrm{Pd}\right)$ & $59-84 \%$ & 3 \\
\hline $\mathrm{JLNU}-4$ & $64 \mathrm{wt} \%$ & 4 \\
\hline SBMOF-1 and SBMOF-2 & $22.6 \%$ and $42.7 \%$ & 5 \\
\hline$\left[\mathrm{Zn}_{3}(\mathrm{BTC})_{2}\left(\mathrm{H}_{2} \mathrm{O}\right)_{3}\right]$ & $20 \%$ & This work \\
\hline
\end{tabular}

HIna $=$ isonicotinic acid, (JLNU-4; JLNU = Jilin Normal University; $\mathrm{H}_{4} \mathrm{ABTC}=3,3^{\prime}$,5,5' azobenzenetetracarboxylic acid) [Cd(ABTC)(H2O)2(DMA)] 4DMA, SBMOF-1 [Ca(sdb); sdb: 4,4'-sulfonyldibenzoate],SBMOF-2 [Ca(tcpb); tcpb: 1,2,4,5-tetrakis(4-carboxyphenyl)- benzene] 
Table S5. Photocatalytic Degradation of Aqueous RhB and MB by some previously reported MOFs.

\begin{tabular}{|l|l|l|l|l|l|}
\hline \multicolumn{1}{|c|}{ MOF Catalyst } & \multicolumn{1}{|c|}{ Dye } & Efficiency(\%) & Time(min) & $k\left(\mathrm{~min}^{-1}\right)$ & \multicolumn{1}{c|}{ Ref. } \\
\hline & & & & & \\
\hline $\mathrm{Co}_{3}(\mathrm{BPT})_{2}(\mathrm{bpp})$ & RhB & 90 & 120 & 0.0192 & 4 \\
\hline MIL-53(Fe) & RhB & 98 & 40 & 0.0794 & 6 \\
\hline$\left[\mathrm{Co}(\mathrm{bpba})(\mathrm{bdc})_{1 / 2}\right]$ & MB & 84 & 180 & - & 7 \\
\hline NNU-36 & RhB & 96 & 70 & 0.0468 & 8 \\
\hline Cd-PDA & MB & 85 & 120 & 0.0153 & 9 \\
\hline MIL-88(Fe) & MB & 100 & 50 & - & 10 \\
\hline MIL-88(Fe $)$ & RhB & 100 & 60 & - & 10 \\
\hline$\left[\mathrm{Co}_{2}(1,4-\mathrm{BDC})(\mathrm{NCP})_{2}\right]$ & RhB & 68 & 300 & - & 11 \\
\hline$\left[\mathrm{Co}_{2}(1,4-\mathrm{BDC})(\mathrm{NCP})_{2}\right]$ & MB & 63 & 300 & - & 11 \\
\hline$\left[\mathrm{Zn}_{3}(\mathrm{BTC})_{2}\left(\mathrm{H}_{2} \mathrm{O}\right)_{3}\right]$ & RhB & 85 & 90 & 0.0095 & This work \\
\hline$\left[\mathrm{Zn}_{3}(\mathrm{BTC})_{2}\left(\mathrm{H}_{2} \mathrm{O}\right)_{3}\right]$ & MB & 79 & 90 & 0.0084 & This work \\
\hline
\end{tabular}

$\mathrm{BPT}=$ biphenyl-3,4',5-tricarboxylic acid; bpp = 1,3-bis(4-pyridyl)propane; Hbpba = 3,5bis(pyridin-4ylmethoxy)benzoic acid; $\mathrm{H}_{2}$ bdc $=1$,4-benzenedicarboxylate; $\mathrm{H}_{2} \mathrm{PDA}=$ 9-phenylcarbazole-3,6-dicarboxylic acid; 1,4- $\mathrm{H}_{2} \mathrm{BDC}=$ benzene-1,4-dicarboxylic acid; $\mathrm{HNCP}=$ 2-(4-carboxyphenyl)imidazo(4,5-f)(1,10)phenanthroline).

\section{References}

1. Arıcı, M.;Yeşilel,O. Z.;Tasand,M.;Demiral, H. Effect of Solvent Molecule in Pore for Flexible Porous Coordination Polymer upon Gas Adsorption and Iodine Encapsulation, Inorg. Chem., 2016, 55, 1303513038.

2. Liu, J-H.; Qi, Y-J.; Zhao, D.; Li,H-H.; Zheng, S-T.Heterometallic Organic Frameworks Built from Trinuclear Indium and Cuprous Halide Clusters: Ligand-Oriented Assemblies and Iodine Adsorption BehaviorInorg. Chem., 2019, 58, 516 -523.

3. Massasso, G.; Castillo, M. R.; Long, J.; Haines,J.; Vinot, S.D.; Maurin, G.; Grandjean, A.; Onida,B.; Donnadieu,B.; Larionova, J.; Guérin, C.; Guari, Y. Molecular iodine 
adsorption within Hofmann-type structures $\mathrm{M}(\mathrm{L})\left[\mathrm{M}^{\prime}(\mathrm{CN})_{4}\right]\left(\mathrm{M}=\mathrm{Ni}, \mathrm{Co} ; \mathrm{M}^{\prime}=\mathrm{Ni}, \mathrm{Pd}\right.$, Pt): impact of their composition,Dalton Trans., 2015, 44, 19357-19369.

4. Yao, C.; Wang, W.; Zhang, R.S.; Li, H-Y.; Xu, Y-H.; Su, Z-M.; Che, G-B. A multifunctional microporous metal-organic framework: efficient adsorption of iodine and column-chromatographic dye separation, $R S C A d v$., 2018, 8, 36400-36406.

5. Banerjee, D.; Chen, Xianyin.; Lobanov, S. S.; Plonka, A. M.; Chan, X.; Daly, J. A.; Kim, T.; Thallapally, P. K.; Parise,Iodine Adsorption in Metal Organic Frameworks in the Presence of Humidity., J. B. ACS Appl. Mater. Interfaces, 2018, 10, 10622-10626.

6. Ai, L.; Zhang, C.; Li, L.; Jiang, J.Iron terephthalate metal-organic framework: Revealing the effective activation of hydrogen peroxide for the degradation of organic dye under visible light irradiation, Appl. Catal.B, 2014, 148-149, 191-200.

7. Zhao, J.; Dong, W. W.; Wu, Y. P.; Wang, Y. N.; Wang, C.; Li, D. S.; Zhang, Q. C. J. Mater. Chem. A., 2015, 3, 6962-6969.

8. H. Zhao, Q. Xia, H. Xing, D. Chen and H. Wang, Construction of Pillared-Layer MOF as Efficient Visible-Light Photocatalysts for Aqueous $\operatorname{Cr}(\mathrm{VI})$ Reduction and Dye Degradation,ACS Sustainable Chem. Eng., 2017, 5, 4449-4456.

9. Wu, P.; Liu, Y.; Li, Y.; Jiang, M.; Li, X.; Shi Y.; Wang, J. A cadmium(ii)-based metalorganic framework for selective trace detection of nitroaniline isomers and photocatalytic degradation of methylene blue in neutral aqueous solution J. Mater. Chem., A, 2016, 4, 16349-16355.

10. Wu, Y.; Luo, H.; Wang, H. Synthesis of iron(iii)-based metal-organic framework/graphene oxide composites with increased photocatalytic performance for dye degradation, RSC Adv., 2014, 4, 40435-40438.

11. Sun, H.-Y.; Liu, C.-B.; Cong, Y.; Yu, M.-H.; Bai, H-Y.; Che, G.-B. New photocatalyst for the degradation of organic dyes based on $\left[\mathrm{Co}_{2}(1,4-\mathrm{BDC})(\mathrm{NCP})_{2}\right]_{\mathrm{n}} \cdot 4 \mathrm{nH}_{2} \mathrm{O}$ Inorg. Chem. Commun., 2013, 35, 130-134. 